\title{
INTEGRAÇÃO DE MÍDIAS DIGITAIS NO ENSINO FUNDAMENTAL: EXPERIÊNCIAS COM A LEITURA PROTOCOLADA
}

Leila Laís Gonçalves ${ }^{1}$

\author{
Graziela Fatima Giacomazzo²
}

\begin{abstract}
Resumo. Este artigo apresenta as reflexões sobre o desenvolvimento e aplicação de uma ferramenta interativa para suporte ao processo de leitura protocolada e indicativos sobre as potencialidades do uso de mídias digitais como recurso educacional. O estudo é resultado das atividades elaboradas e realizadas no âmbito de um projeto de extensão. Para tanto, apresentam-se os conceitos de Leitura protocolada, inferências e mídia digital. Situa-se metodologicamente como pesquisa-ação no contexto do Ensino Fundamental de uma escola municipal. Os desafios a serem superados para a integração e a produção das mídias digitais no ensino ainda são muitos. Parte deles se relacionam com o reduzido conhecimento tecnológico dos professores e a falta de uma cultura digital para o uso pedagógico dos recursos. Para isso, faz-se necessário ampliar a formação de professores e o desenvolvimento de estratégias para a inserção das mídias digitais nos processos de ensino e aprendizagem.
\end{abstract}

Palavras-chave: Mídias Digitais, Leitura Protocolada, Ensino Fundamental.

\section{INTEGRATION OF DIGITAL MEDIA IN FUNDAMENTAL EDUCATION: EXPERIENCES WITH PROTOCOLED READING}

\begin{abstract}
This article presents the reflections on the development and application of an interactive tool to support the process of reading protocol and indicative about the potential of the use of digital media as an educational resource. The study is the result of the activities elaborated and carried out in the scope of an extension project. For that, the concepts of protocol reading, inferences and digital media are presented. It is located methodologically as action research in the context of the Elementary School of a municipal school. The challenges to be overcome for the integration and production of digital media in education are still many. Some of them relate to the reduced technological knowledge of teachers and the lack of a digital culture for the pedagogical use of resources. To do this, it is necessary to expand teacher training and the development of strategies for the insertion of digital media in teaching and learning processes.
\end{abstract}

Keywords: Digital Media, Protocoled Reading, Elementary Education.

\footnotetext{
${ }^{1}$ Mestre em Ciência da Computação. Docente e Pesquisadora na Universidade do Extremo Sul Catarinense. Ilg@unesc.net

2 Doutora em Educação. Docente e Pesquisadora na Universidade do Extremo Sul Catarinense. gfg@unesc.net
}

Criar Educação, Criciúma, v. 8, nº1, jan/jul 2019.- PPGE - UNESC 


\section{Introdução}

As constantes inovações das tecnologias de informação e comunicação (TIC) e a disseminação do uso das mídias digitais vêm consolidando a cultura midiática e exercendo forte influência na formação dos indivíduos (TERUYA, 2006; FELIPE; TERUYA, 2009). A linguagem da mídia está presente no cotidiano, no trabalho, na escola e com papel fundamental no processo de comunicação em seus múltiplos formatos verbais e não-verbais (imagem, som, movimento, texto, interação).

Apesar da relevância e da presença da mídia digital nos diferentes contextos, o seu uso ainda é pouco explorado como um recurso efetivo e significativo no processo de ensino e aprendizagem. Dentre as principais dificuldades no uso de mídias na educação, cita-se: 1) pouca habilidade dos professores na utilização dos recursos tecnológicos; 2) ausência de planejamento didático de forma sistemática que considere as especificidades dos conteúdos e 0 suporte das mídias; 3) pouco uso das mídias como ferramenta e linguagem para ensinar e aprender; 4) insuficiência na infraestrutura e manutenção dos recursos físicos necessários; 5) não delineamento de estratégias de uso (OLIVEIRA, 2011; BACICH; MORAN, 2015).

Considerando significativo o uso das mídias digitais em sala de aula e as condições dos professores na sua utilização, entende-se que a formação de professores integrada à realidade escolar pode suprir as necessidades emergentes em relação ao conhecimento teórico e prático necessário para a integração das mídias na educação. Esse artigo apresenta os resultados de uma das etapas de um projeto de extensão universitária realizado em uma escola municipal. O projeto teve como objetivo produzir, integrar e efetivar o uso de mídias digitais e recursos interativos nos processos pedagógicos visando a melhoria dos níveis de alfabetização e potencializando a inclusão digital na comunidade escolar. Participaram do projeto professores e acadêmicos dos cursos de Pedagogia, Artes Visuais e Ciência da Computação da Universidade do Extremo Sul Catarinense (Unesc). 
O eixo de aplicação dessa etapa do projeto foi a leitura, indicado pela escola como prioritário nos dois primeiros anos do ensino fundamental. Uma das questões apontadas pelos professores foi a necessidade do desenvolvimento da habilidade de fazer inferências sobre um tema ou deduzir informações implícitas em um texto utilizando a leitura literária como meio para abordagem das diferentes unidades temáticas, objetos de conhecimento e habilidades preconizados pela Base Nacional Comum Curricular (BNCC) (BRASIL, 2018). Partindo do pressuposto que as práticas de leitura devem propiciar uma postura de interação ativa do aluno-leitor entendeu-se que a estratégia de leitura protocolada ou pausa protocolada pode auxiliar no desenvolvimento da inferência quando utilizada de forma consciente e sistematizada em sala de aula. Buscando ampliar as possibilidades da estratégia de leitura protocolada, optou-se pelo uso das tecnologias digitais no desenvolvimento de recursos de suporte à prática da leitura que propiciassem a interação multimidiática e multimodal.

O uso de tecnologia digital tem propiciado meios interativos de leitura, buscando atender novas habilidades e necessidades dos usuários. O uso de mídias digitais e da interatividade vêm provocando mudanças nos suportes e estilos de leitura e invocando inovações em relação ao livro impresso. Recursos como: imagens e textos interativos, animações, vídeos, sons e intervenções diretas no texto, hiperligações e entrelaçamento de conteúdos a partir de links tem sugerem uma relação diferenciada entre o suporte e o leitor, por possuir características como a imaterialidade e a fragmentação (PAIVA, 2015). Dentre os desafios, na proposição de interatividade na leitura, estão a definição de estratégias para apresentação do conteúdo de forma coerente, diferenciada e interessante ao leitor e que possa contribuir efetivamente com os objetivos pedagógicos de inferência desejados na prática da leitura. Outro desafio é o desenvolvimento técnico da proposta exigindo soluções baseadas em fundamentos teóricos e metodológicos envolvendo design de interação de de experiência de uso.

Nesse sentido, foi desenvolvida a ferramenta interativa "Lendo, fazendo e aprendendo" para mediar a aplicação da estratégia de leitura protocolada do livro de literatura, "As tranças de Bintou" de Sylviane Anna Diouf, Criar Educação, Criciúma, v. 8, nº 1, jan/jul 2019.- PPGE - UNESC 
nos segundos anos do ensino fundamental da Escola Municipal de Educação Infantil e Ensino Fundamental Hercílio Amante de Criciúma/SC.

O artigo está estruturado a partir desta introdução, na seção 2 são apresentados os conceitos de leitura protocolada e o uso da intervenção e habilidade de inferência no processo, e mídia digital. O caminho metodológico do estudo e o desenvolvimento da ferramenta interativa são descritas na seção 3; a seção 4 traz os resultados da experiência de aplicação da ferramenta apontando os indicativos sobre as potencialidades do uso de mídias digitais como recurso educacional e as conclusões são apresentadas na seção 5.

\section{Leitura protocolada e as práticas de inferências}

Inferir informações implícitas é uma habilidade fundamental para se compreender um texto; exige que o leitor associe conhecimento linguístico a conhecimento extralinguístico, o que caracteriza leitores experientes, que conseguem ir além das informações encontradas na superfície textual, requerendo desses leitores um nível de leitura mais avançado do que a etapa inicial de decodificação. Para realizar inferências, o leitor deve conjugar, no processo de produzir sentidos para o que lê, as pistas oferecidas pelo texto aos seus conhecimentos prévios, à sua experiência de mundo. Estão envolvidas na construção da competência de fazer inferências, as habilidades de: inferir o sentido de uma palavra ou expressão a partir do contexto no qual ela aparece; inferir o sentido de sinais de pontuação ou outros recursos morfossintáticos; inferir uma informação a partir de outras que o texto apresenta ou, ainda, o efeito de humor ou ironia em um texto (BRASIL, 2008).

A leitura protocolada ou pausa protocolada é uma técnica de leitura em etapas que envolve a produção de inferências fazendo com que haja uma interação entre aluno, texto e autor (COSCARELLI, 1996). Nas inferências, ou pontos de parada, a leitura é interrompida de forma planejada para que o leitor (aluno) possa rever, compreender e refletir sobre o que foi lido articulando seu repertório com as informações. Ao utilizar esta técnica de leitura, o professor 
pode propiciar situações interativas buscando a construção de sentido na leitura por meio das inferências e ultrapassando a visão de leitura como mera extração de informações (FARIAS, 2012).

Neste caso usa-se a mídia digital, que se refere ao conjunto de meios e dispositivos de comunicação, baseados em tecnologia digital, que possibilitam a comunicação ou distribuição digital de produções escritas, sonoras ou visuais (LEMOS, 2008). A integração das mídias digitais no ensino visa apoiar o processo de aprendizagem, sendo um recurso imprescindível o uso dos diferentes meios na melhoria da qualidade do ensino. É importante que a escola diversifique a forma de apresentação dos conteúdos oferecendo formatos multimidiáticos e digitais. Para isso, é importante o direcionamento do uso dos meios e ferramentas para que educadores e educandos se tornem-se agentes coparticipantes (SCHALCH; MELO, 2017). Nesse processo, é imprescindível que haja a compreensão e incorporação de novas linguagens e seus modos de funcionamento, aplicando-as como possibilidades de comunicação e aprendizagem participando de forma consciente da disseminação da cultura digital.

De acordo com a BNCC (BRASIL, 2018), é possível propiciar uma vivência significativa e situada, ao se envolver gêneros verbais e híbridos potencializados pelos meios digitais. $O$ uso de gêneros escritos e multimodais variados a partir de práticas situadas contribuem para o desenvolvimento da leitura crítica e para a construção de um percurso criativo e autônomo de aprendizagem da língua. A apresentação de situações de leitura organizadas em pré-leitura, leitura e pós-leitura pode potencializar a aprendizagem na perspectiva de um (re)dimensionamento das práticas e competências leitoras já existentes, especialmente em língua materna (BRASIL, 2018).

A interatividade, compreendida como diferentes possibilidades de atuação (forma e nível de influência) que o meio propicia ao usuário, amplia o impacto educacional da mídia digital (MUSSOI; TAROUCO, 2011). Atuando como uma estratégia importante na aprendizagem significativa. A interatividade pode ser aplicada em atividades com ações de seleção, organização, integração de 


\section{(190)}
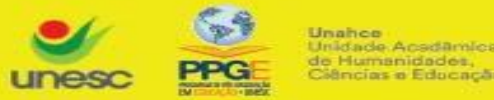

informações agregando e abrangendo questões de motivação, conhecimento prévio e habilidades de uso de mídia (TAROUCO et al., 2009).

\section{Caminho metodológico}

Os procedimentos adotados para a execução do projeto enquadram-se nos parâmetros da pesquisa-ação (GIL, 2002), considerando o ponto de partida uma necessidade real no contexto escolar e como caminho a busca de possibilidades para encontrar possibilidades a partir da investigação bibliográfica, planejamento de ações e intervenção na prática dos professores participantes com o intuito de aprimorá-la. Com o propósito de ser descritiva e de natureza aplicada, buscou-se abordar, de forma quali-quantitativa, a trajetória do desenvolvimento do trabalho apresentando a necessidade, a reflexão teórica sobre o assunto, a proposta de intervenção e os resultados obtidos com a experiência a partir do olhar dos envolvidos.

Participam do projeto as três turmas do segundo ano do ensino fundamental da Escola Municipal de Educação Infantil e Ensino Fundamental Hercílio Amante de Criciúma/SC, as três professoras das turmas e o professor de informática da escola. A seleção das turmas ocorreu por adesão das professoras. Foram setenta crianças com idade entre sete e oito anos.

As etapas realizadas foram: 1) planejamento dos procedimentos para integração das mídias digitais nas práticas pedagógicas; 2) modelagem do aplicativo "Lendo, fazendo e aprendendo" identificando as interações e a forma de inserção das mídias; 3) desenvolvimento da ferramenta e das mídias; 4) testes e instalação de softwares de apoio no laboratório de informática; e 5) acompanhamento de uso.

O planejamento e validação dos procedimentos para integração das mídias digitais foram realizados em conjunto com os professores. A proposta de intervenção com uso da estratégia de leitura protocolada teve como ponto de partida o livro de literatura, "As tranças de Bintou" de Sylviane Anna Diouf. A definição das paradas teve como foco as unidades temáticas, os objetos de Criar Educação, Criciúma, v. 8, nº 1, jan/jul 2019.- PPGE - UNESC 

conhecimento, ritmo de aprendizagem e o interesse dos alunos. Para cada parada foram definidos os objetivos, temas abordados, interações, atividades, mídias e links com outros componentes curriculares e temas transversais. Foram planejadas dez paradas para a realização de interações visando desenvolver habilidades de inferência: 1) o título; 2) a capa; 3) a contracapa; 4) a autora; 5) o ilustrador; 6) identidade: o eu e a família 7) autoimagem, autoestima e sonhos; 8) a cultura: etnia, costumes, comidas e tradições; 9) os lugares: a paisagem, transporte; e 10) valores: o eu e os outros. Em cada parada, as inferências envolveram a identificação de informações implícitas e explícitas no texto e o uso das mesmas para ampliar a compreensão, reforçar conteúdos estudados, aumentar vocabulário, desenvolver novos aprendizados, conectar outros conhecimentos, exercitar os temas e assuntos correlacionados e aguçar a curiosidade para a leitura. As mídias foram planejadas, produzidas e integradas para dar suporte eficiente à comunicação da mensagem considerando o público infantil, o contexto educativo, as informações e as questões semióticas de sentido e significado. As mídias digitais utilizadas na comunicação foram imagens, vídeos, textos, áudios e recursos interativos.

O desenvolvimento da ferramenta interativa "Lendo, fazendo e aprendendo" foi efetuado por acadêmicos do curso de Ciência da Computação, bolsistas do projeto de extensão. As tecnologias utilizadas na implementação da ferramenta foram: Axure, HTML5, CSS 3 e API JavaScript. Para a concepção e desenvolvimento da aplicação foram considerados três fatores, que de acordo com Behar (2009) são fundamentais em materiais digitais para fins didático: o fator gráfico, o fator técnico e o fator pedagógico. Para atender o fator gráfico foi considerada a função da imagem nas interfaces e sua relação com a aprendizagem. Quanto ao fator técnico, o foco foi o planejamento da navegação e usabilidade visando suporte na jornada do interator nas interfaces da ferramenta. No fator pedagógico, buscou-se como base as teorias de aprendizagem e sua aplicação com uso de estratégias na concepção do conteúdo e desenvolvimento do design de interação e de experiência de uso (UX). O design de UX é uma arte e ciência que, unindo todos os elementos que compõe os sistemas interativos, 
visa prover (SAFFER, 2009): 1) as necessidades dos usuários, suas limitações, objetivos, desejos e expetativas; 2) os objetivos dos produtores do conteúdo em relação aos resultados com os usuários; e 3) a integração de todas as partes, entendendo que o todo é a soma de cada parte.

A ferramenta foi disponibilizada em mídia flexível (pen drive ou DVD) e instalada nos computadores do laboratório de informática da escola. Para a utilização de recursos envolvendo edição de texto, busca de conteúdo Web e desenho digital foram instalados softwares de apoio nos computadores do laboratório de informática. Foram realizados testes de funcionamento da ferramenta verificando compatibilidade com sistema operacional (Windows 7 ou superior e Linux educacional 4 ou superior).

\section{Aplicação da Ferramenta "Lendo, fazendo e aprendendo"}

Uma importante contribuição da etapa do projeto de extensão, relatada nesse artigo, foi o desenvolvimento da ferramenta interativa "Lendo, fazendo e aprendendo" para a leitura protocolada do livro de literatura "As tranças de Bintou". A figura 1 mostra telas da ferramenta.
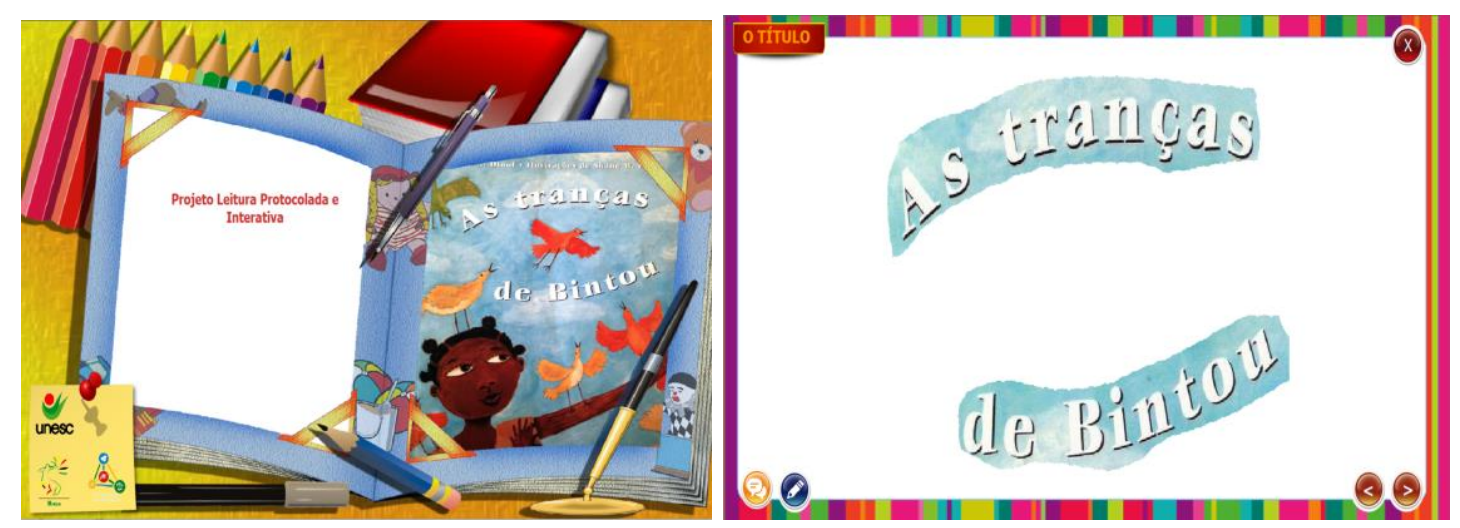

Figura 1. Telas: inicial e da parada 1 - 0 título

A interação e navegação na ferramenta é realizada com o recurso de toque, mouse ou teclado com os ícones seta para esquerda e seta para direita. Foram utilizados ícones para identificar as possibilidades de interação em cada Criar Educação, Criciúma, v. 8, nº1, jan/jul 2019.- PPGE - UNESC 
parada. O ícone de balões de comunicação aparece sempre que há inferência em relação a informações implícitas e explícitas. Quando acionado este ícone, é apresentada a tela com as mídias para a comunicação da inferência (figura 2)

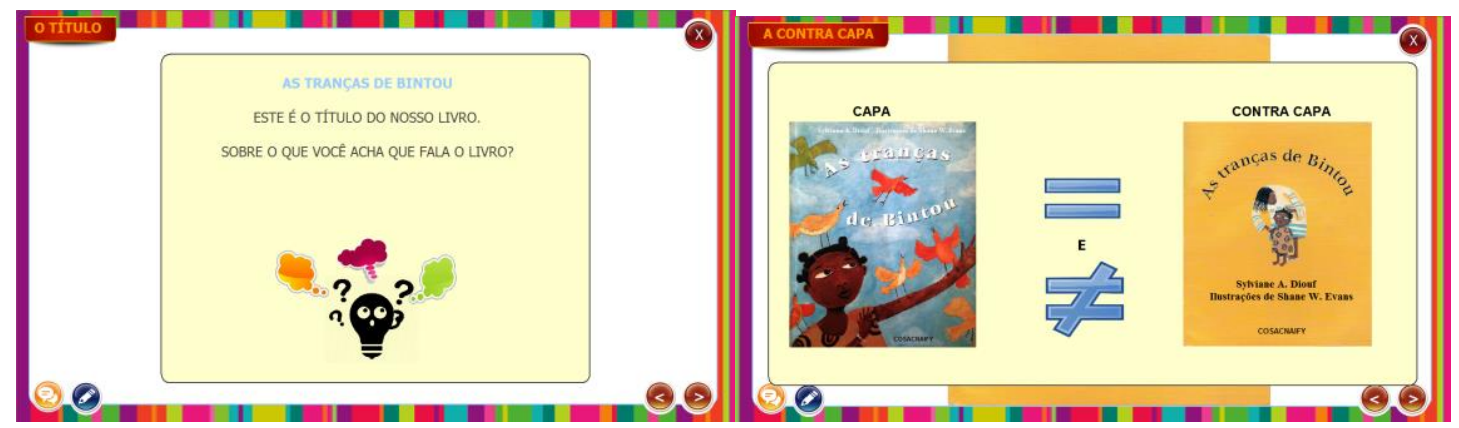

Figura 2. Telas de inferência das paradas: 1 - 0 título e 3 - A contracapa

O ícone lápis foi aplicado para indicar atividades a serem desenvolvidas. Ao clicar nesse ícone, o usuário pode receber uma instrução para executar uma ação fora da ferramenta (figura 3 - A) ou uma atividade que deverá ser executada na ferramenta a partir de interações como cliques, seleção de áreas, ações de arrastar objetos, digitação ou toque (figura 3 - B).

A

B
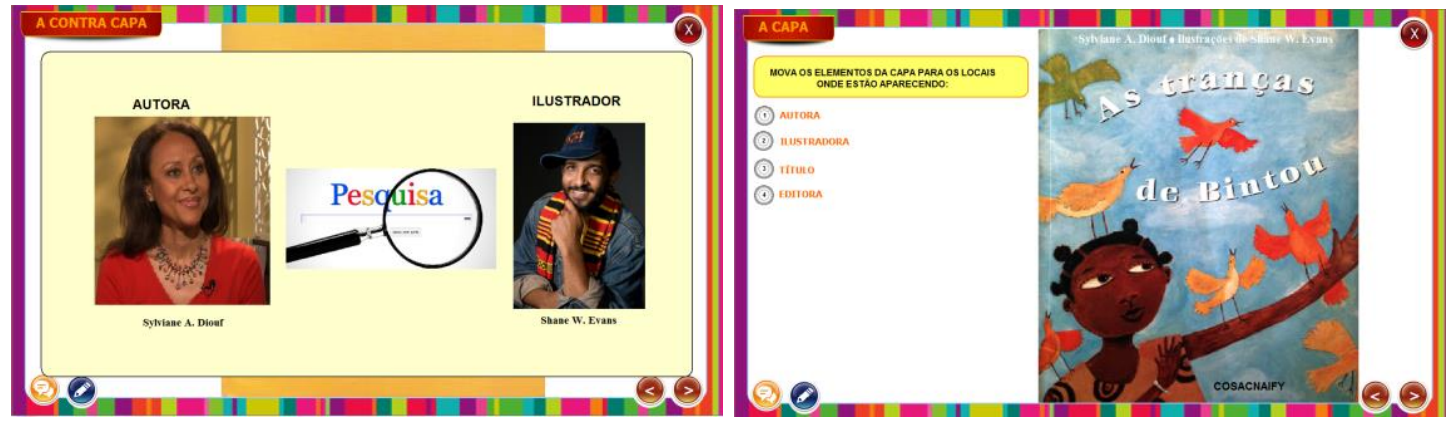

Figura 3. Tela de atividades das paradas: 3 - A contracapa e 2 - A capa

A aplicação da ferramenta interativa possibilitou identificar indicativos sobre as potencialidades do uso de mídias digitais no processo de aprendizagem e reafirmar a contribuição das mesmas para a diversificação da forma de apresentação dos conteúdos em formatos multimidiáticos e digitais. O local de aplicação foi no laboratório da Escola Municipal de Educação Infantil e Ensino 
Fundamental Hercílio Amante de Criciúma/SC. Participaram dessa ação três turmas de segundo ano do ensino fundamental, em um total de setenta crianças, acompanhadas das professoras de sala de aula e do laboratório de informática. Para cada turma (em torno de 23 alunos) foram realizadas dez intervenções com duração de uma hora e intervalos de uma semana entre elas. As intervenções foram executadas e acompanhadas por professores e acadêmicos participantes do projeto de extensão.

Como desdobramentos das intervenções, planejadas nas paradas da leitura protocolada, foi possível ampliar o uso de mídias digitais como recurso pedagógico. Uma das atividades propostas foi a edição de imagem tendo como resultado uma fotomontagem composta pela foto do aluno caracterizado com indumentária da etnia negra, abordada na leitura (figura 4).

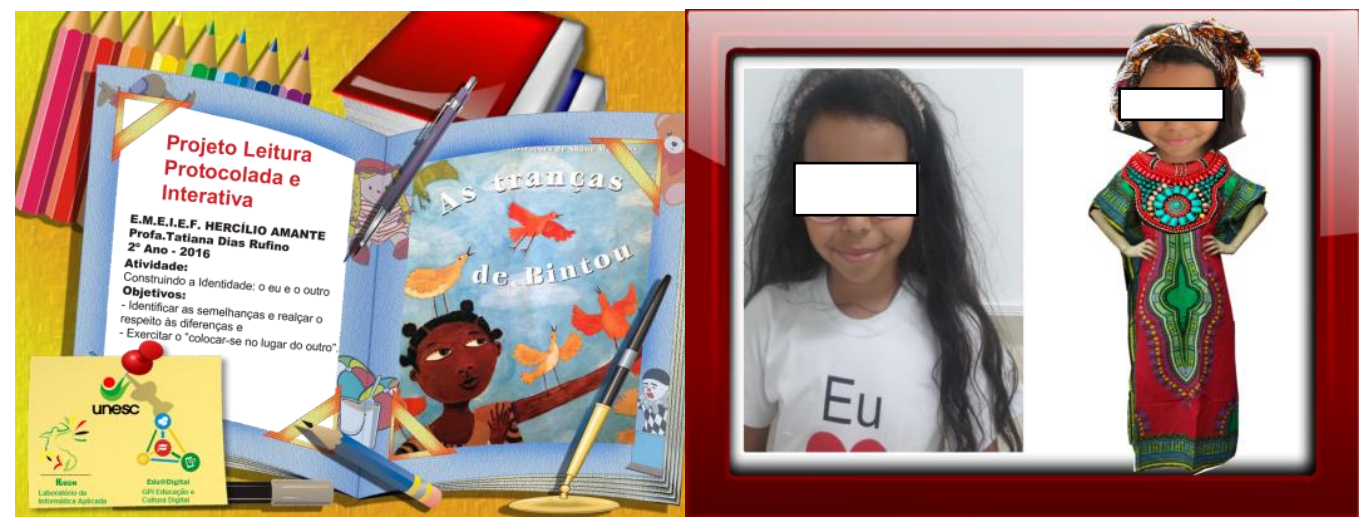

Figura 4. Tela da parada 8 - A cultura e atividade de fotomontagem

A partir das intervenções com o uso do aplicativo, das observações e do relato dos alunos e professores, identificou-se algumas contribuições possibilitadas pela linguagem audiovisual, ou seja, bauso de imagens, vídeos, textos, áudio e interações. Os indicativos sobre as potencialidades do uso de mídias digitais como recurso educacional mais significativos foram: 1) resultados melhores na compreensão do texto; 2) conexões com outros elementos do repertório dos alunos no momento da leitura; 3) ampliação do processo de interpretação e significação das informações; 4) interação entre os conteúdos e 5) inclusão digital em atividades curriculares. 


\section{Considerações finais}

Com experiência foi possível também confirmar a necessidade da preparação dos professores para sua incorporação no processo de ensino e aprendizagem e constatar a importância do seu envolvimento no processo. Foi visível e verbalizado o entusiasmo dos professores no projeto diante das possibilidades de ampliação da compreensão da leitura e da melhoria dos níveis de alfabetização dos seus alunos. Outros resultados das ações são os reflexos que incidem na escola apontando uma aproximação cada vez maior entre a universidade e a comunidade.

Os desafios a serem superados para a integração e a produção das mídias digitais no ensino ainda são muitos. Parte deles se relacionam com o reduzido conhecimento tecnológico dos professores e a falta de uma cultura digital para o uso pedagógico dos recursos. Para isso, faz-se necessário ampliar a formação de professores e o desenvolvimento de estratégias para a inserção das mídias digitais nos processos de ensino e aprendizagem.

\section{Referências}

BACICH, Lilian; MORAN, José. Aprender e ensinar com foco na educação híbrida Revista Pátio, № 25, junho, 2015, p. 45-47. Disponível em <http://www2.eca.usp.br/moran/wp-content/uploads/2015/07/hibrida.pdf >. Acesso em 14 mar. 2019.

BEHAR, Patrícia. Modelos Pedagógicos em Educação a Distância. São Paulo: Artmed Editora, 2009.

BRASIL. Ministério da Educação. Base Nacional Comum Curricular: Ensino Médio. Brasília: MEC/Secretaria de Educação Básica, 2018.

BRASIL. Ministério da Educação. PDE: Plano de Desenvolvimento da Educação: Prova Brasil: ENSINO FUNDAMENTAL: matrizes de referência, tópicos e descritores. Brasília: MEC, SAEB; INEP, 2008.

COSCARELLI, Carla Viana. O ensino da leitura: uma perspectiva psicolinguística. In: Boletim da Associação Brasileira de Linguística. Maceió: Imprensa Universitária, dez.1996. p. 163-174. 
FARIAS, Dui Barroso Lima. O processo tradutório e a construção do sujeito no discurso. Universidade Federal do Rio de Janeiro. Faculdade de Letras Dissertação de Mestrado. Rio de Janeiro, 2012.

FELIPE, Delton Aparecido; TERUYA, Teresa Kazuco. Imagens da África no cinema: o potencial da mídia no ensino de história. ETD - Educação temática Digital, Campinas, v.11, n.1, p.96 -122, 2009.

GIL, Antonio Carlos. Como elaborar projetos de pesquisa. 4a ed. São Paulo: Atlas; 2002. p. 41-57.

LEMOS, André. Cibercultura: Tecnologia e vida social na cultura contemporânea. Porto Alegre: Sulina, 2008.

MUSSOI, Eunice Maria; TAROUCO, Liane Margarida Rockenbach. Interatividade com objetos de aprendizagem. Cadernos de Informática (UFRGS), v. 6, p. 297-300, 2011.

OLIVEIRA, Natascha Borba. Mídia-educação: uma discussão sobre a abordagem das mídias no contexto escolar. Monografia - Departamento de Comunicação, Faculdade de Biblioteconomia e Comunicação, Porto Alegre, RS, 2011.

PAIVA, Marília Meireles de. Meu Quarto: Livro Interativo Infantil para dispositivos móveis. Relatório técnico. Departamento de Desenho Industrial. Universidade de Brasília, UNB, 2015. Disponível em <http://bdm.unb.br/bitstream/10483/10907/1/2015_MariliaMeirelesDePaiva.pdf>. Acesso em 10 mar. 2019.

SAFFER, Dan. Designing for Interactions. New Riders. 2009.

SCHALCH, Cynthia Stelita; MELO, Maria Cecília Mendonça. A utilização de recursos de mídias no processo de ensino aprendizagem: identificação de novos perfis docentes. Universidade Tecnológica Federal do Paraná - Curitiba Paraná - n.15, p. 18-33, 2015. Disponível em <http://revistas.utfpr.edu.br/pb/index.php/revedutec-ct/article/view/1921 >. Acesso em 10 mar. 2019.

TAROUCO, Liane Margarida Rockenbach; et al. Multimídia Interativa: princípios e ferramentas. RENOTE: revista novas tecnologias na educação [recurso eletrônico]. Porto Alegre, v. 7, n. 1, jul. 2009. Disponível em <http://seer.ufrgs.br/renote/article/view/14014/7904>. Acesso em 10 mar. 2019.

TERUYA, Teresa Kazuco. Trabalho e educação na era midiática. Maringá, PR: Eduem. 2006. 